MATEC Web of Conferences 51, 01012 (2016)

DOI: $10.1051 /$ matecconf/20165101012

(C) Owned by the authors, published by EDP Sciences, 2016

\title{
Design and Numerical Analysis of Spiral Pressure Recovery Blades in the Overflow Pipe of the Cyclone Separator
}

\author{
Qidong Chen ${ }^{1, a}$, Yuegao Sun ${ }^{2}$ \\ ${ }^{1}$ Changshu institute of Technology, Changshu, 215500; \\ ${ }^{2}$ Soochow University, Suzhou, 215000
}

\begin{abstract}
Spiral pressure recovery blades which can make the rotational flow convert into direct flow in overflow pipe of the cyclone separator are designed in the overflow pipe. The numerical calculation of the cyclone separator with different blades is carried out by using the Reynolds stress turbulence (RSM) model. Meanwhile, the trend of the total pressure of the inlet and outlet of the cyclone separator is discussed while the blades are inserted at different positions. Results show that the pressure of inlet and overflow outlet obviously reduced when the blade is inserted in the cyclone separator. It can make the inlet total pressure reduced more than $9 \%$ and the overflow outlet total pressure reduced more than $50 \%$ when four blades are inserted in the cyclone separator. The total pressure of the inlet and overflow outlet is the smallest when blades are inserted in the lowest of the overflow pipe.
\end{abstract}

\section{Introduction}

At present, the cyclone separator has been widely used in many fields, such as paper, coal, mineral processing, food, petroleum, chemical industry and so on. Many experts and scholars have done a lot of research on the performance of the cyclone separator. The influence of the pressure field and velocity field of the cyclone separator was studied by Jiang Minghu et al[1]. Wang Zunce et al [2] studied the influence of different inlet structure parameters of the cyclone separator on the pressure loss. The flow field of the cyclone separator was studied by Chen Qidong et al [3]. The influence of operation parameters on the separation performance of cyclone separator has also been studied and tested by many experts and scholars [4]. However, the study on the pressure recovery in the overflow pipe is relatively few. The fluid in the overflow pipe is a high speed rotational flow which has considerable rotational kinetic energy. If it is converted into direct flow, the mechanical friction loss can be reduced and dynamic pressure is converted to static pressure, and thus the pressure of the inlet and outlet of the cyclone separator is reduced.

In this paper, a new type of pressure recovery blade which can change the rotational flow into direct flow is designed. The influence of three different kinds of blade number on the inlet and outlet pressure is compared and the best position of the blade inserted into the overflow pipe is discussed. ${ }^{\text {a }}$

\section{Design of pressure recovery blade}

${ }^{a}$ Corresponding author: 18015565894@sina.cn 


\section{MATEC Web of Conferences}

\section{1 theoretical analysis}

In order to convert rotational flow into direct flow, the attack angle of the front inlet edge of the blade must be maximally as the same as the attack angle for the fluid to enter the blade. Through the numerical calculation for the flow field of the cyclone without blades, Fig. 1 shows 10 fluid tracks $0.045 \mathrm{~m}$ away from the overflow outlet in the overflow pipe. According to the figure, the motion tracks of the fluid are not regular cylindrical spiral curves and the spiral distance thereof is constantly changed, so these tracks cannot be expressed by the standard spiral curve equation.

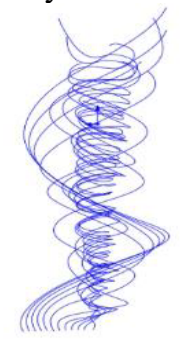

Figure 1.The track curves of the overflow pipe.

In the paper, the finite division theory is adopted to divide the whole fluid track into infinite small arcs which can be approximated into standard cylindrical spiral curves. The lower endpoint of an independent track is recorded as $f_{0}(x, y, z)$ and the upper endpoint is recorded as $f_{n}(x, y, z)$, and the endpoints of an optional arc taken from the above track are respectively recorded as $f_{a}(x, y, z)$ and $f_{a+1}(x, y, z)$. Meanwhile, the horizontal plane of point $f_{a}(x, y, z)$ is assumed as plane $\alpha$, the projection of point $f_{a+1}(x, y, z)$ in plane $\alpha$ is assumed as $f_{a+1}(x, y, z) \alpha$ and the projection of the above arc in plane $\alpha$ is assumed as $f_{a} f_{a+1 \alpha}$. Since the arc is very small, the length thereof can be replaced by line $\overline{f_{a} f_{a+1}} \alpha$. Subsequently, the lead angle of the spiral curve can be calculated according to Formula (1) for the lead angle of the standard cylindrical spiral curve.

$$
\tan \varphi_{a}=\frac{z_{a+1}-z_{a}}{f_{a} f_{a+1} \alpha}=\frac{z_{a+1}-z_{a}}{\sqrt{\left(x_{a+1}-x_{a}\right)^{2}+\left(y_{a+1}-y_{a}\right)^{2}}}
$$

Similarly, central angle can be approximately calculated according to Formula (2).

$$
\theta_{\alpha}=\frac{2 \overline{f_{a} f_{a+1}}}{O_{\alpha} f_{a}+O_{\alpha} f_{a+1 \alpha}}=\frac{2 \sqrt{\left(x_{a+1}-x_{a}\right)^{2}+\left(y_{a+1}-y_{a}\right)^{2}}}{\sqrt{x_{a}^{2}+y_{a}^{2}}+\sqrt{x_{a+1}^{2}+y_{a+1}^{2}}}
$$

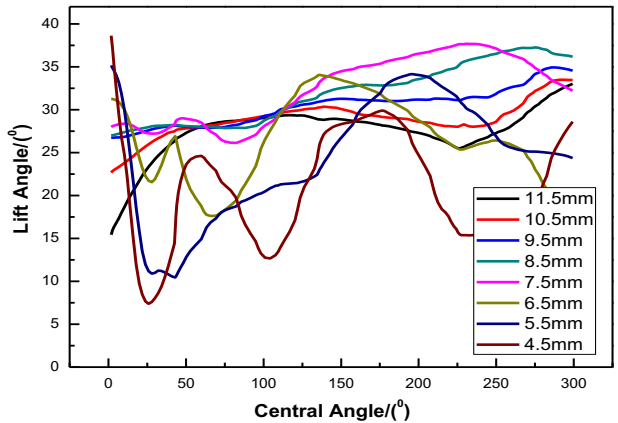

(a) Lift angle changes with the central

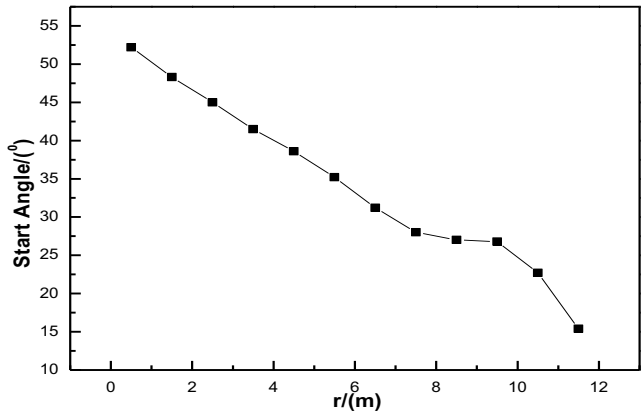

(b)Initial lift angle change of 10track curves with $r$

Figure 2. Lift angle changes with the center angle and initial angle with the radial position change

After the coordinates of the division point of the track are exported, Formulae (1) and (2) can be adopted to calculate the lead angle and the central angle corresponding to each small arc. The variation curve of central angle along with lead angle is as shown in Fig.2(a). According to the 
figure, when $\theta$ is in the range of $0^{\circ} \sim 30^{\circ}$, the lead angles of the tracks have a stable variation tendency, wherein the lead angles of the circumjacent tracks (for example $r=11.5 \mathrm{~mm}, 10.5 \mathrm{~mm}$, $9.5 \mathrm{~mm} ; r$ refers to the radial position) have linear increase tendency while the lead angles of the tracks near the central axis (for example $r=4.5 \mathrm{~mm}, 5.5 \mathrm{~mm}$ ) have reduction tendency. Additionally, the variation curves of the initial lead angles of 10 tracks at the same height and along the same radial direction along with radial position $r$ are as shown in Fig.2(b). According to the figure, the initial lead angles have obvious reduction tendency. Therefore, when $\theta$ is in the range of $0^{\circ} \sim 30^{\circ}$, the track curves are variable spiral surfaces. According to the above analysis, the spiral pressure recovery blade is reasonable.

\subsection{Design of blade}

In Solidworks software, 10 fluid tracks exported by the post-processing software are taken as the boundary, and then the boundary surface command is adopted to generate the front surface of the blade, and then the front surface is connected to the vertical plane through the transition surface, thus to establish the whole blade model. Specifically, the boundary dimension of the blade is as follows: the height of the whole blade is $30 \mathrm{~mm}$, the blade thickness is $0.5 \mathrm{~mm}$, the leading central angle of the bottom blade is $30^{\circ}$, the blade radius is $11.5 \mathrm{~mm}$, and the initial lead angle of the blade is gradually increased from the outside to the inside, with the variation range of $15^{\circ} \sim 90^{\circ}$. The pressure recovery blade models with different blade quantities are as shown in Fig.3.

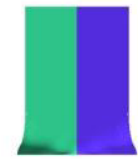

(a)two blades
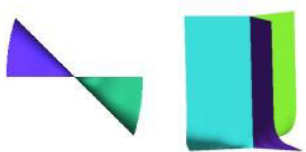

(b)three blades

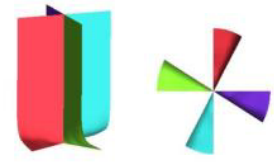

(c)four blades

Figure 3. Different blade models.

\section{Numerical calculation model}

Without isotropy and eddy viscosity hypotheses, Reynolds stress model[5] can be used to simulate such complex three-dimensional motion as strong vortex flow. Liang Zheng, et al. [6] have comprehensively explained why Reynolds stress model is more applicable to the simulation of the internal flow field of the cyclone separator than other models (for example, standard $k-\varepsilon$ model[7] and RNG $k-\varepsilon$ model[8]). Specifically, ICEM software is adopted for hexagonal structure meshing, and the computational domain of each model is about 500,000 mesh points, and the hexagonal structure mesh diagrams of the five different models are as shown Fig. 4.
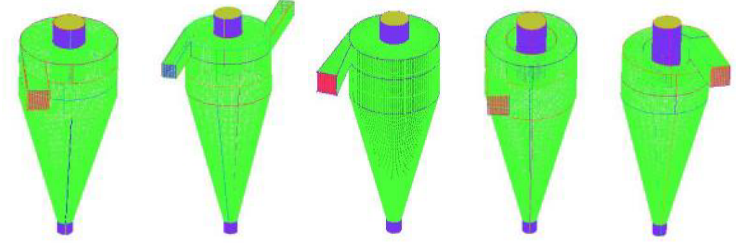

Figure 4. grid graph of different models.

Meanwhile, FLUENT software is adopted to calculate the internal flow field of the cyclone, and the velocity field and pressure field coupled SIMPLEC algorithm is adopted as the calculation method for transient calculation, wherein the time step is $0.001 \mathrm{~s}$ and the residual error control value is set as 0.00001. Additionally, the pressure item is PRESTO algorithm and the second-order upwind scheme is adopted for other equations.

\subsection{Geometrical physical model}


Classic $75 \mathrm{~mm}$ cyclone is adopted in the paper, wherein Model I is the single-inlet rectangular tangent feed pipe cyclone, Model II is the dual-inlet rectangular tangent feed pipe cyclone, Model III is the shrinkable rectangular tangent feed pipe cyclone, Model IV is the concentric annular feed pipe cyclone, Model V is the three-dimensional spiral feed pipe cyclone, and the structural parameters of the above five models[9] are as shown in Table 1. $D$ is the diameter of the cylindrical barrel, $a$ and $b$ are respectively the length and the height of the rectangular inlet, $h$ is the height of the cylindrical barrel, $\alpha$ is the taper angle, $d_{o}$ is the diameter of the underflow port, $d_{u}$ is the diameter of the overflow outlet, $d$ is the diameter of the concentric annulus and $z$ is the spiral distance.

Table 1. Structural parameters of five models.

\begin{tabular}{llllllllll}
\hline & $\begin{array}{c}D \\
(\mathrm{~mm})\end{array}$ & $\begin{array}{l}a \\
(\mathrm{~mm})\end{array}$ & $\begin{array}{l}b \\
(\mathrm{~mm})\end{array}$ & $\begin{array}{l}h \\
(\mathrm{~mm})\end{array}$ & $\begin{array}{l}\alpha \\
\left({ }^{\circ}\right)\end{array}$ & $\begin{array}{l}d_{o} \\
(\mathrm{~mm})\end{array}$ & $\begin{array}{l}d_{u} \\
(\mathrm{~mm})\end{array}$ & $\begin{array}{l}d \\
(\mathrm{~mm})\end{array}$ & $\begin{array}{c}z \\
(\mathrm{~mm})\end{array}$ \\
\hline Model I & 75 & 17 & 27 & 75 & 20 & 12.5 & 25 & & \\
Model II & 75 & 12 & 19 & 75 & 20 & 12.5 & 25 & & \\
Model III & 75 & 17 & 27 & 75 & 20 & 12.5 & 25 & 40 & \\
Model IV & 75 & 17 & 27 & 75 & 20 & 12.5 & 25 & & 17 \\
Model V & 75 & 17 & 27 & 75 & 20 & 12.5 & 25 & & \\
\hline
\end{tabular}

\subsection{Boundary conditions}

1) The velocity inlet is adopted as the inlet, and the inlet velocity is set as $2.28 \mathrm{~m} / \mathrm{s}$.

2) The pressure outlets are adopted as the overflow outlet and the underflow outlet, the static pressure is set as 0 and is communicated with the atmosphere.

3) No-slip boundary is adopted for the wall surface, and the standard wall function is adopted for the wall treatment.

4) Physical property parameters of the mixture: the water density is $998 \mathrm{~kg} / \mathrm{m}^{3}$ and the viscosity is $0.001 \mathrm{Pas}$.

\section{Reliability analysis of results}

American scholar Hsieh [10] adopted the laser Doppler tachymeter to measure and obtain the velocity distributions on different sections of a cyclones as early as 1988. Many experts and scholars[11-13] have adopted the above experimental data to verify the accuracy of the calculation results. In the paper, the post-processing software is adopted to output the radial position based distributions of the tangential velocity (Fig.5(a)) and the axial velocity (Fig.5(b)) at the position $60 \mathrm{~mm}$ away from the highest point of the cylindrical barrel, and meanwhile such distribution data are analyzed and compared with Hsieh's experimental data.

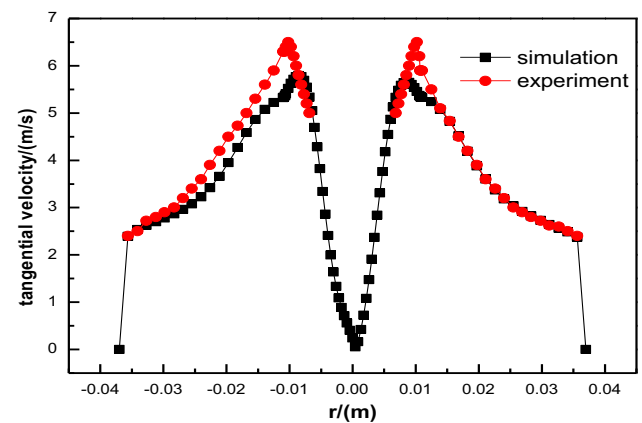

(a) Tangential velocity with radial position distribution

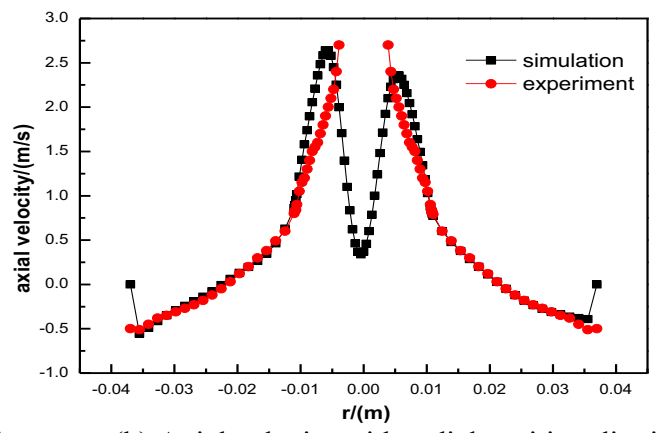

(b) Axial velocity with radial position distribution

Figure 5. Comparison of tangential velocity and axial velocity between numerical simulation and experiment 
According to the figure, the simulation result is basically consistent with the experimental data; the simulation experiment has wall error, because the wall boundary is not accurately processed in the numerical simulation and it is difficult to accurately measure the tangential velocity and the axial velocity at the position near the central position in the experiment.

\section{Flow field analysis}

\subsection{The velocity field and pressure field of Model I under different blade number}

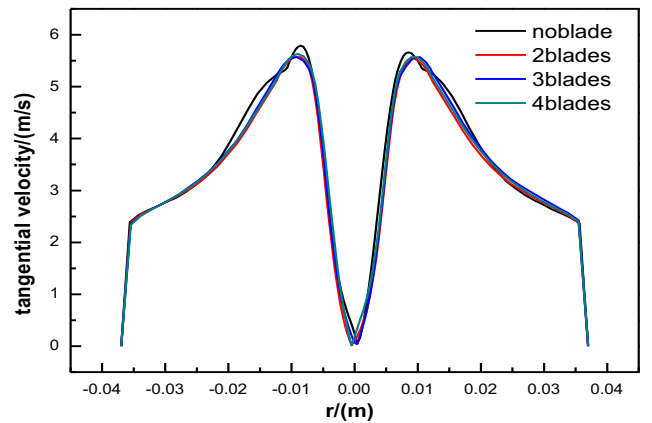

(a)

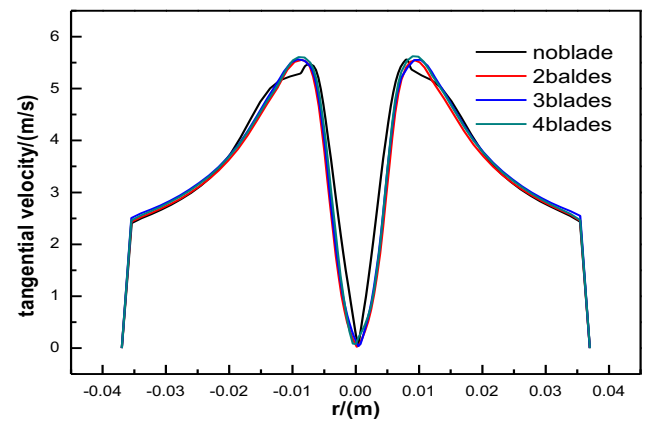

(c)

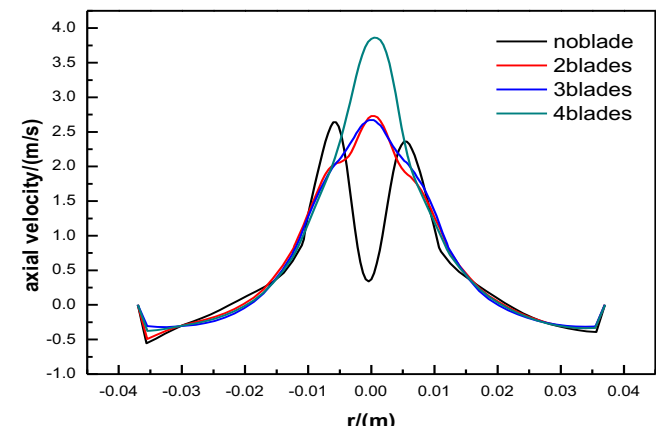

(b)

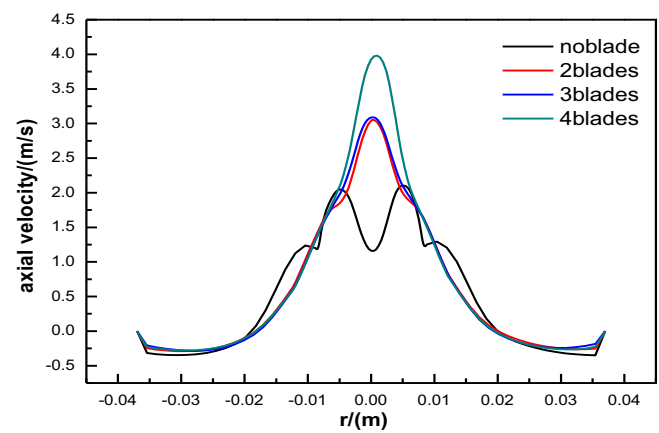

(d)

Figure 6. The radial distribution of axial velocity and tangential velocity in different blade models (from top to bottom: $z=60 \mathrm{~mm} 、 z=75 \mathrm{~mm})$

The radial position based variation tendency of the axial velocity and the tangential velocity at the cross sections $Z=60 \mathrm{~mm}$ and $Z=75 \mathrm{~mm}$ in Model I with different blade quantities is as shown in Fig.6. According to the figure, the tangential velocity curves all present a dual-peak shape, and the downward concave of the tangential velocity curve is caused by the wall thickness of the overflow pipe of the cyclone, and the peak values of the tangential velocity almost appear at the same position, thus verifying Liang Zheng's research achievement: the maximum tangential velocity appears at the radial position slightly more than $2 r_{0} / 3\left(r_{0}\right.$ is the diameter of the overflow pipe). According to the tangential velocity distribution diagram, in the forced vortex area, the tangential velocity of the cyclone inserted with blades is less than that of the cyclone not inserted with blades, thus indicating that the pressure recovery blades can weaken the rotating strength of the forced vortex and reduce the total energy for driving the equal fluid as well as reduce the total inlet pressure and the total outlet pressure; the tangential velocity curves of the cyclones respectively inserted with two, three and four blades are basically coincided with each other. According to the axial velocity distribution diagram, in the forced vortex area, the effect of the three-blade cyclone is similar to that of the two-blade cyclone; 
the axial velocity of the four-blade cyclone is more than those of two-blade and three-blade cyclones, so the total outlet pressure of such cyclone can be effectively reduced.

\subsubsection{Pressure fields of Model I with different blades}
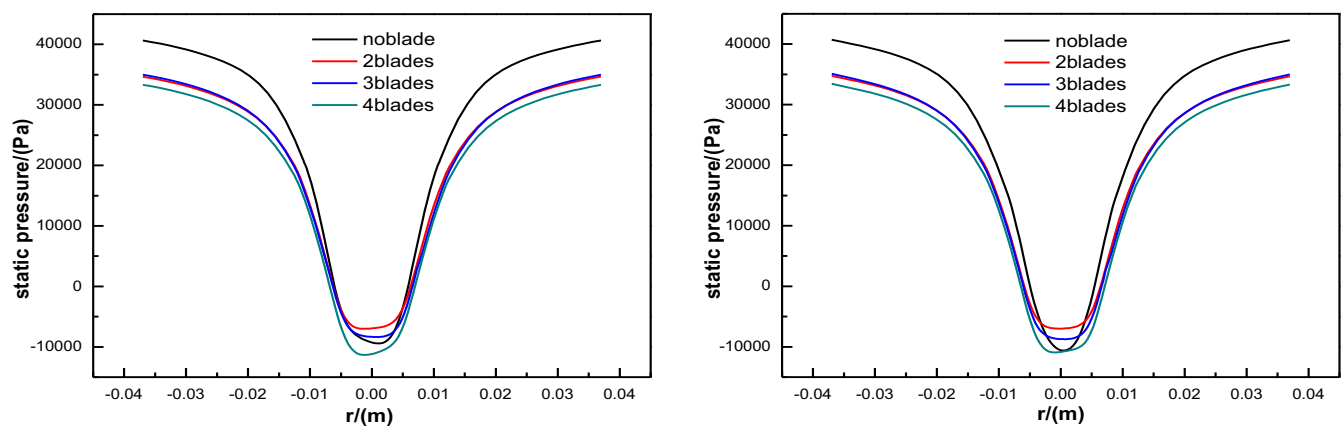

Figure 7. Static pressure distribution at different cross sections of the model 1 (from left to right: section $\mathrm{z}=60 \mathrm{~mm} 、 \mathrm{z}=75 \mathrm{~mm}$ )

Under the conditions of respectively inserting two, three and four blades, the radial position based variation tendency of the static pressure of Model $\mathrm{I}$ at the cross sections $Z=60 \mathrm{~mm}$ and $Z=75 \mathrm{~mm}$ is as shown in Fig.7. According to the figure, the internal pressure of the cyclone separator is basically symmetrically distributed and the static pressure at the same cross section is gradually reduced from the wall surface to the central axis along the radial position. Since the central fluid is under high-speed revolution, thus the negative pressure generated in the central axis area is exactly one of the preconditions for generating air column inside the cyclone. The two curves for cross sections $Z=60 \mathrm{~mm}$ and $Z=75 \mathrm{~mm}$ are basically coincided with each other, thus indicating that the radial pressure gradient on different sections are almost the same. The static pressures at the positions with the same radius are the same, thus indicating that the axial pressure gradient is almost zero. After the pressure recovery blades are inserted, the static pressure in the standard free vortex area is obviously reduced. Additionally, the two-blade cyclone and the three-blade cyclone have equivalent static pressure reduction effect, and the four-blade cyclone can maximally reduce the static pressure, thus having the best effect.

\subsection{Analysis of pressure fields of the blades at different heights in the overflow pipe}

Inlet pressure and outlet pressure are important performance parameters of a cyclone. Under the condition of not influencing the separation efficiency, it is significant to reduce inlet pressure and outlet pressure. Three-blade pressure recovery blades are respectively inserted at five positions in the overflow pipes of the five models, wherein the five positions are respectively $5 \mathrm{~mm}, 15 \mathrm{~mm}, 25 \mathrm{~mm}$, $35 \mathrm{~mm}$ and $45 \mathrm{~mm}$ away from the overflow outlet surface. FLUENT software is adopted to calculate the total inlet pressure and the total overflow outlet pressure of the cyclone separator after the blades are inserted at the above five positions.

The total inlet pressure and the total overflow outlet pressure at each position in the five models are as shown in Fig.8. (Fig.8(a) shows the total inlet pressures of Models I, II, IV and V, Fig.8(b) shows the total inlet pressure of Model III, Fig.8(c) shows the total overflow outlet pressures of Models I, II, IV and V, and Fig.8(d) shows the total overflow outlet pressure of Model III). According to the figure, along with the increase of the insertion depth, the total inlet pressure and the total overflow outlet pressure of the cyclone separator are almost linearly reduced. When the bottoms of the inserted blades and the overflow pipe are at the same horizontal line, more rotational flows are 
converted into direct flows, thus minimizing the total inlet pressure and the total outlet pressure of the cyclone separator and realizing the best effect of the cyclone.

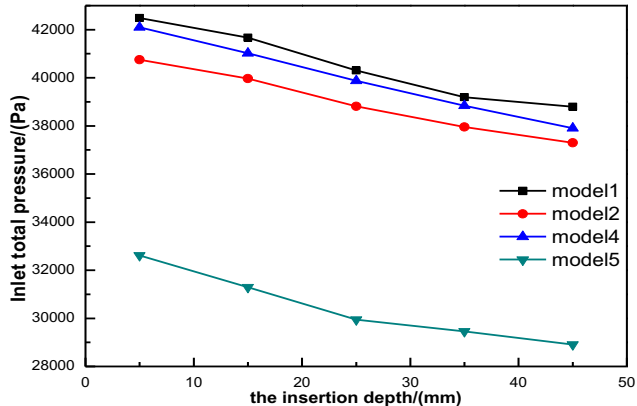

(a)

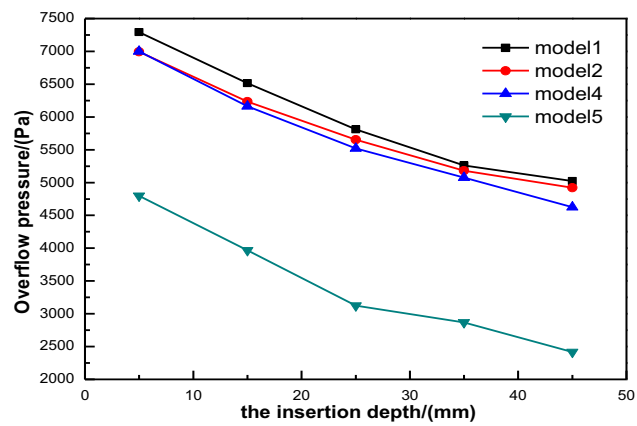

(c)

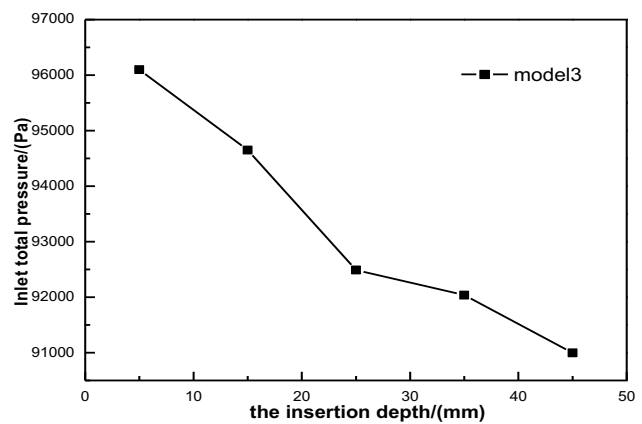

(b)

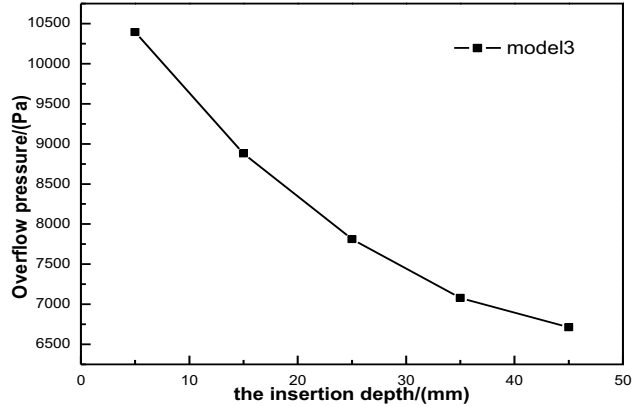

(d)

Figure 8. Relationship between blade insertion depth and total pressure of inlet and overflow outlet

\subsection{Pressure fields of five different models with different blades}

In order to realize the best effect of the cyclone, the best installation position of the pressure recovery blade shall be set at the nethermost end of the overflow pipe. The specific effects of the three blade structures installed at the nethermost ends of the overflow pipes in the five different models will be analyzed and discussed in this section.

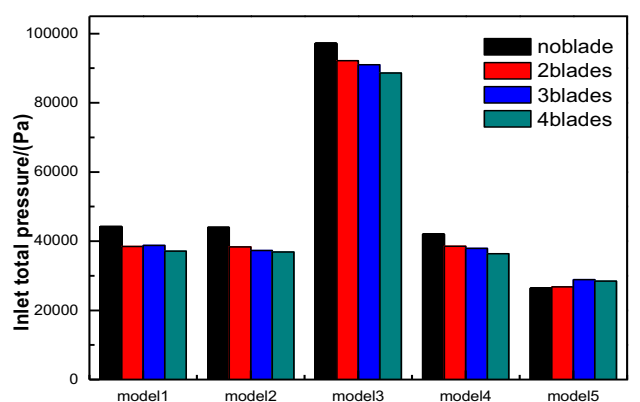

(a) Total inlet pressures

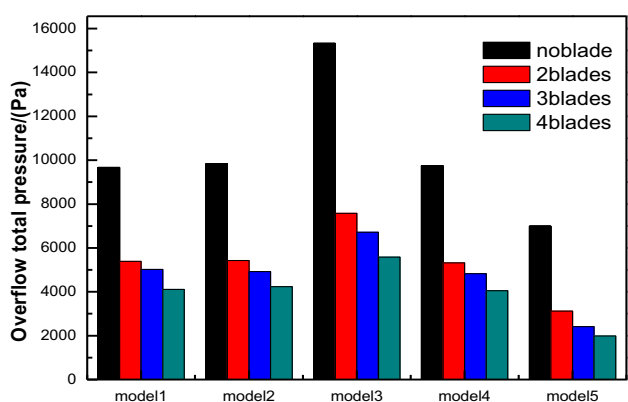

(b) Total overflow outlet pressure

Figure 9. Inlet total pressure and overflow outlet pressure under five different models in different blade

The total inlet pressure and the total outlet pressure of the five different models with different blades are as shown in Fig.9. According to Fig.9(a), the pressure recovery blades can reduce the total inlet pressures of Models I, II, III and IV. For Models I, II and IV, the inserted blades can reduce the 
total inlet pressure at least by $13 \%$; for Model III, the inserted blades can reduce the total inlet pressure by about $6 \%$; for Model $\mathrm{V}$, the inserted pressure recovery blade cannot reduce the total inlet pressure but increase it. According to Fig.9(b), the inserted pressure recovery blades can significantly reduce the total overflow outlet pressure, the two-blade cyclone can reduce the total outlet pressure by about $50 \%$; the four-blade cyclone has the best effect for reducing the total inlet pressure and the total overflow outlet pressure, and the effect of the three-blade cyclone is only inferior to that of the fourblade cyclone.

\section{Conclusion}

1) The inserted pressure recovery blades can obviously reduce the total inlet pressure and the total overflow outlet pressure of the cyclone. The four-blade cyclone separator can reduce the total inlet pressure by about $9 \% \sim 16 \%$ and reduce the total overflow pressure by about $50 \% \sim 70 \%$.

2) The cyclone separator inserted with the pressure recovery blades at the nethermost end of the overflow pipe has the best effect.

3 ) It is not suitable to insert the pressure recovery blades into the three-dimensional feed pipe cyclone separator.

\section{References}

1. JANG. Ming-hu, LIU. Dao-you, ZHAO. Li-xin, JIA. Gui-chun. The Effect of Cone Angle to Hydrocyclone Pressure Field and Velocity Field[J]. Chemical Machinery, 5:572-576 (2011)

2. WANG. Zun-ce, GAO. Ye, Lu. Feng-xia, LIU. Xiao-ming, LI. Feng, The Effect of Liquid - liquid Hydrocyclone Inlet Structure Parameters to Pressure Characteristics[J]. Fluid Machinery, 2:16-19 (2003)

3. CHEN. Qi-dong, ZUO. Zhi-quan, The Flow Field Numerical Analysis of Different Lateral Inlet Cyclone[J]. China Engineering Science, 2:58-67+78 (2014)

4. YUAN. Hui-xin, YU. Jian-feng, LIU. Hong-bin, The Effect of Split Ratio to the Basic Performance of Oil-water Separation Hydrocyclone [J]. Petroleum Machinery, 9: 17-19+2 (2000)

5. ZHANG Shi-shuai. Computational Fluid Dynamics and the Application [M].Wuhan: Huazhong University of Science and Technology Press (2011)

6. LIANG. Zheng, WANG. Jin-jin, REN. Lian-cheng, LI. Lian-ming, The Theoretical Research of Solid-liquid Separation Hydrocyclone Flow Field[M]. Beijing: Petroleum Industry Press, 2:25 (2011)

7. Launder B E, spalding D B, Lectures in Mathematical Models of Turbulence [M]. Academic Press, London, England (1972)

8. Vahot V, Orszag S A. Development of turbulent models for shear flow by a double expansion technique[J].Phys Fluid, 4(7):1510-1520 (1992)

9. ZHAO. Qing-guo, ZHANG. Ming-xian, Separation Technology of Hydroclone [M].Beijing: Chemical industry Press, 53 (2003)

10. K. T. Hsieh. Phenomenological Model of the Hydrocyclone [D].Salt Lake City: The University of Utah(1988)

11. K. W. Chu, B. Wang, A. B .Yu, et, al. CFD -DEM Modelling of Multiphase Flow in Dense Medium Cyclones[J].Powder Technology, 193(3):235-247 (2009)

12. J. A. Delgadillo,R. K. Rajamani. A comparative study of three turbulence-closure models for the hydrocyclone problem [J]. Int. J. Miner. Process, 77:217-230 (2005)

13. XU. Yan-xia. The Numerical Simulation and Analysis of Hydrocyclone Separation Process[D]. East China University of Science and Technology(2012) 